\title{
Strangulation of the Floating Gallbladder by the Lesser Omentum
}

\author{
Woo Seok Kim, Jin Hyung Lee \\ Department of Surgery, Soonchunhyang University Gumi Hospital, Gumi, Korea
}

\begin{abstract}
The strangulation of the gallbladder by the omentum is extremely rare. We report a unique case of acute cholecystitis due to the strangulation of a floating gallbladder by the lesser omentum. A 16-year-old male patient presented to a local hospital after the recurrent epigastric pain. The clinical features and image study suggested the gallbladder torsion, and the patient was referred for surgical treatment. Abdominal computed tomography showed no cholecystolithiasis, but the fundus and body of the gallbladder were markedly swollen with wall thickening, whereas the neck of the gallbladder was normal. Based on these findings, the gallbladder torsion was clinically diagnosed and he underwent laparoscopic cholecystectomy. The narrowed part of the gallbladder was tightly strangulated by the lesser omentum. So, strangulation of a floating gallbladder by the lesser omentum was finally diagnosed. We believe this case to be the third case of reported literatures in English.
\end{abstract}

Keywords: Strangulated gallbladder; Floating gallbladder; Lesser omentum

\section{INTRODUCTION}

A floating gallbladder is characterized by a minute attachment to the liver bed and increased mobility [1]. So, a floating gallbladder can easily twist at the level of attachment to the liver bed. Torsion of the gallbladder is defined as the rotation of the gallbladder on its mesentery along the axis of the cystic duct and artery. Torsion of the gallbladder is an extremely uncommon disease entity with a little over 500 cases described in the literature. Torsion of the gallbladder caused by adhesion, adhesive band, and lesser omentum, not caused by rotation of the gallbladder, are very rarely reported among them. We present the third case of acute cholecystitis due to the strangulation of a floating gallbladder by the lesser omentum.

\section{CASE REPORT}

A 16-year-old otherwise healthy male presented with a 3-week history of intermittent epigastric pain and right upper abdominal discomfort without fever. He had no history of abdominal surgery. On physical examination, mild tenderness was apparent in the epigastric area with no rebound tenderness. Laboratory examina- tion of complete blood cell count and liver function test showed normal findings.

On abdominal computerized tomography, the gallbladder appeared as two separate compartments, without cholecystolithiasis. The narrowed and twisted area was seen between the body and neck of the gallbladder (Fig. 1A). The swollen fundus and body of the gallbladder were located on the ventral side of the gastric antrum, while the neck of the gallbladder appeared normal (Fig. 1B). Torsion of the gallbladder was clinically diagnosed, and the patient was referred for surgical treatment.

Laparoscopy demonstrated that the fundus and body of the gallbladder were firmly distended (Fig. 2A), whereas the neck of the gallbladder was normal (Fig. 2B). The tip of the lesser omentum had wrapped around the border between the body and neck of the gallbladder and tightly strangulated the gallbladder (Fig. 3). Thus, strangulation of a floating gallbladder by the lesser omentum was finally diagnosed. After detachment of wrapped lesser omentum, laparoscopic cholecystectomy for a strangulated gallbladder was not difficult, because the gallbladder was connected to the liver bed only by short mesentery and the neck of the gallbladder was not inflamed. Laparoscopic cholecystectomy was performed successfully. Microscopic examination of the gallblad- 

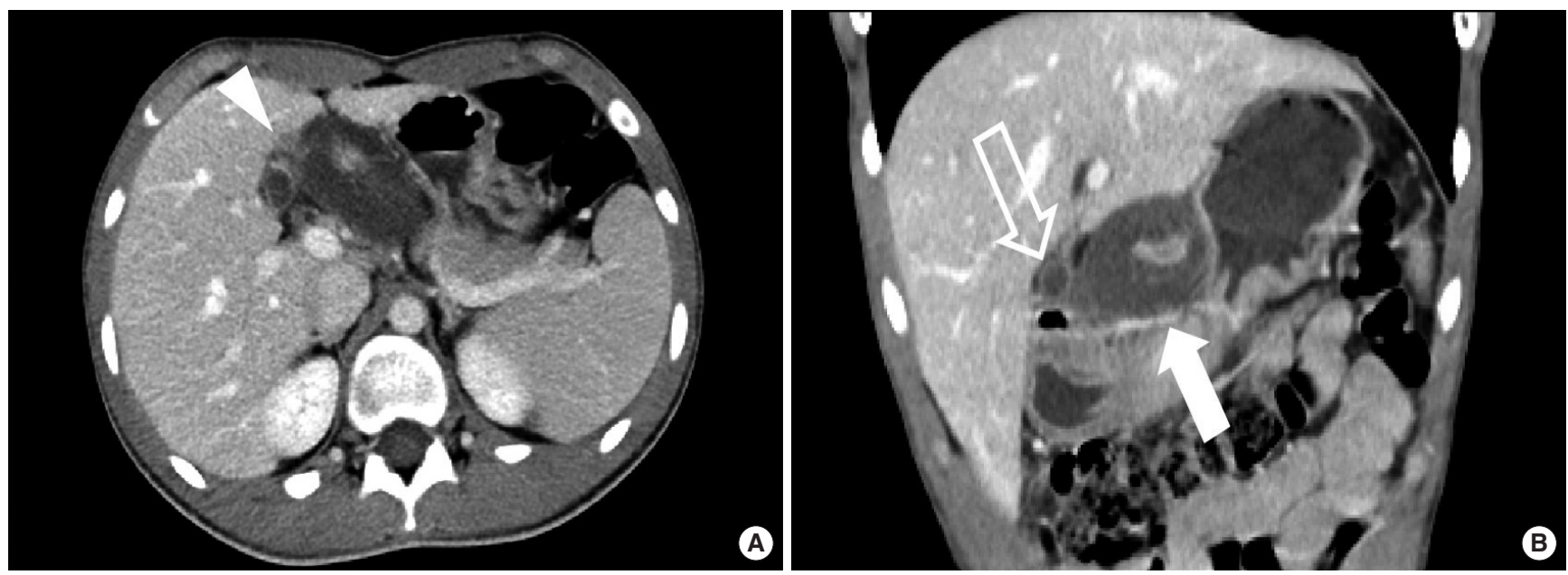

Fig. 1. Abdominal computed tomography images of the gallbladder. (A) The narrowed and twisted area (arrowhead) was seen between the body and neck of the gallbladder. (B) The swollen fundus and body of the gallbladder (white arrow) were located on the ventral side of the gastric antrum, while the neck of the gallbladder (empty arrow) appeared normal.
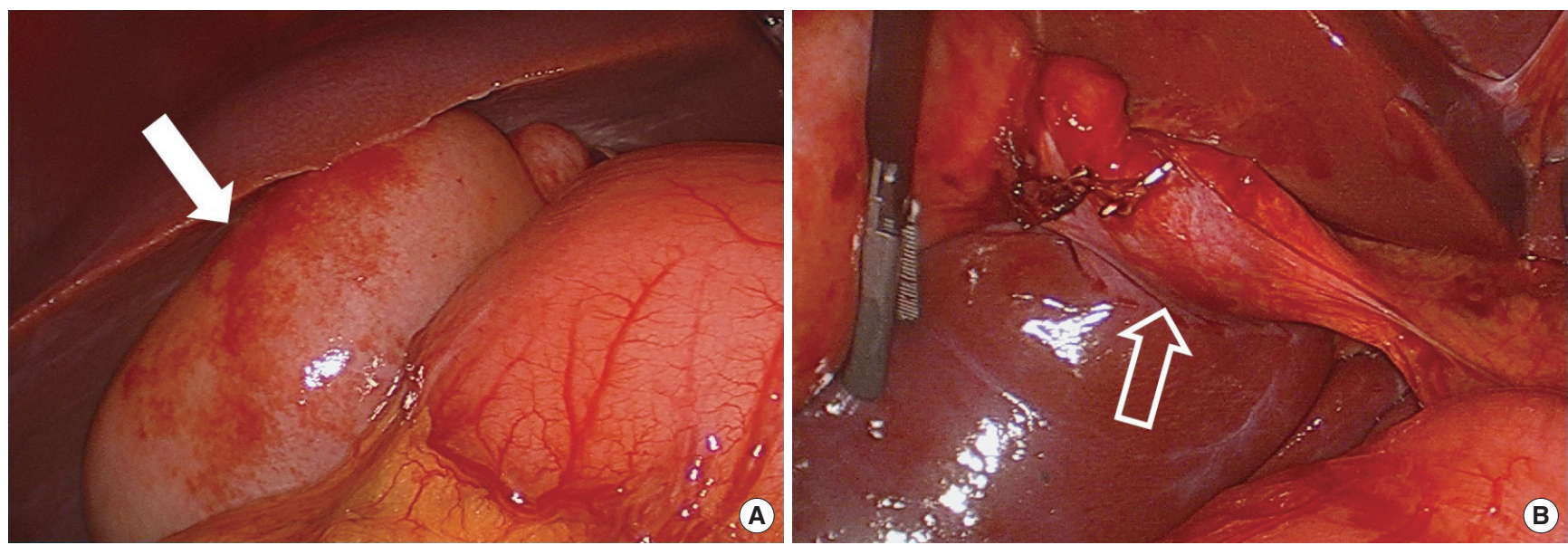

Fig. 2. Intraoperative laparoscopic findings. (A) The fundus and body of the gallbladder (white arrow) were firmly distended. (B) The neck of the gallbladder (empty arrow) was normal.
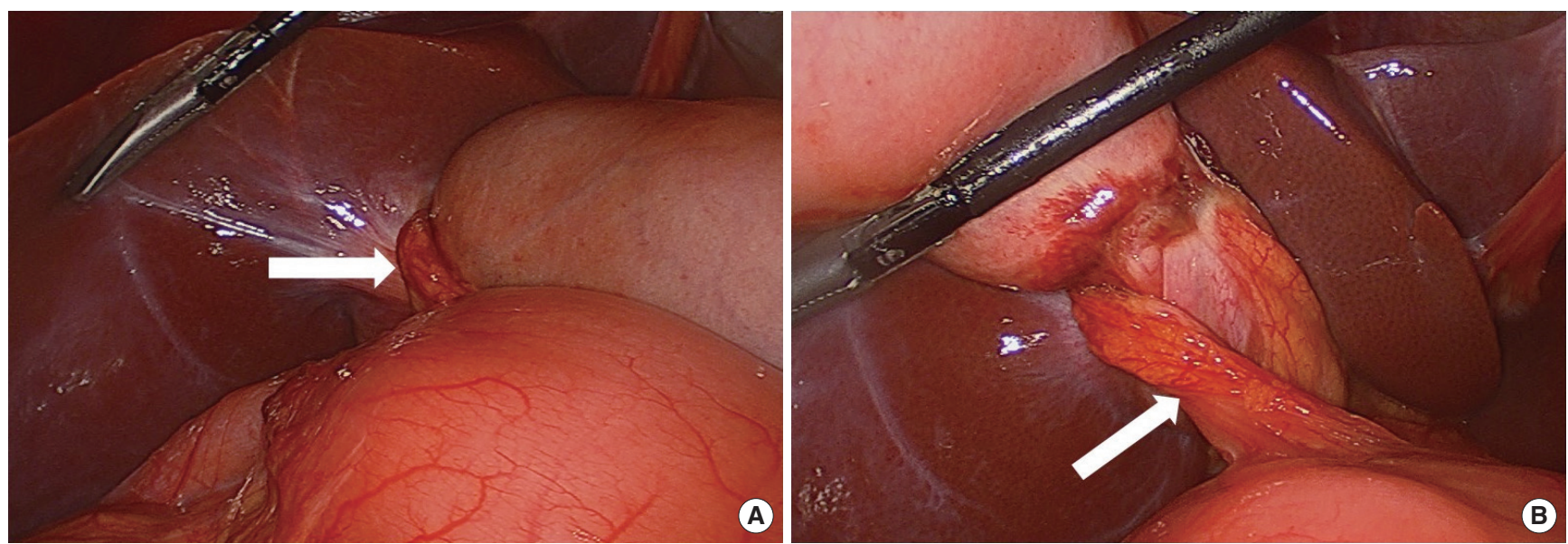

Fig. 3. (A, B) Intraoperative laparoscopic findings. The lesser omentum (white arrow) had encircled around the border between the body and neck of the gallbladder. 


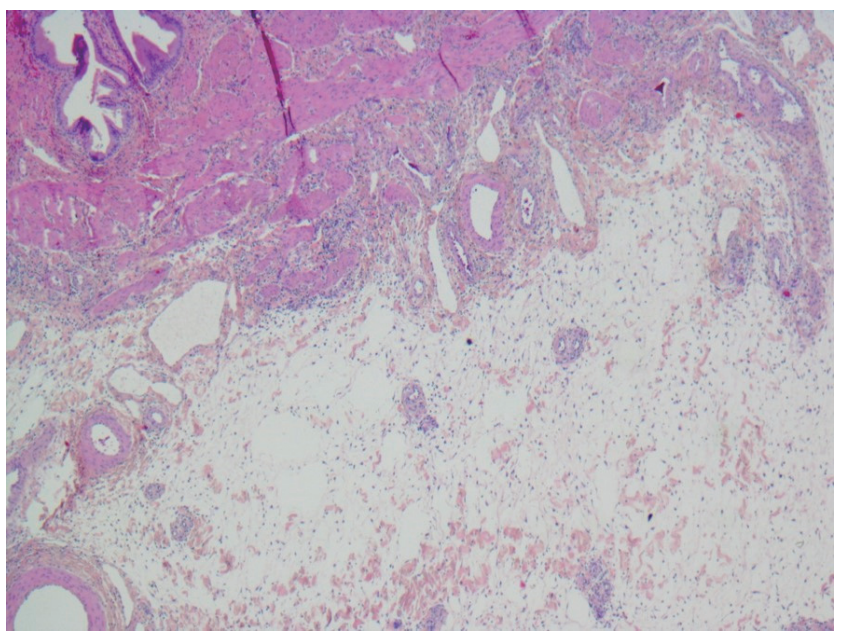

Fig. 4. Low-powered microscopic view of the gallbladder $(\mathrm{H} \& \mathrm{E}, \times 50)$. Microscopic examination disclosed chronic cholecystitis and marked edema of the gallbladder.

der revealed chronic cholecystitis with edematous change (Fig. 4); no stones or tumors were present.

The patient provided written informed consent for the publication of clinical details and images.

\section{DISSCUSSION}

The gallbladder torsion is a rare entity that was first described in 1898 by Wendel [2] in a 25-year-old pregnant woman. Since then, approximately 500 cases have been reported in the literature [3]. However, strangulation of the gallbladder is reported very rarely. To the author's knowledge, only two cases were reported by Ueo et al. [4] and Miyakura et al. [5], and this is the second reported case of strangulation of the gallbladder by the lesser omentum.

Authors report a floating gallbladder with redundant mesenteric attachments as a predisposing factor for torsion [6]. The floating gallbladder can easily rotate at the level of the cystic artery and cystic duct. This rotation causes interruption of its arterial supply and bile flow, which is responsible for the clinical appearance. Thickening of the gallbladder wall, hydrops, and gangrene develop respectively by the torsion of the gallbladder. In our patient, it is possible that repeat episodes of cholecystitis caused by torsion of the floating gallbladder might have resulted in adherence of the tip of the lesser omentum to the gallbladder. This tip of the lesser omentum can be stretched by the movement of the floating gallbladder and become stringy and wound around the gallbladder. Therefore, it is possible that both the floating gallbladder and the lesser omentum can contribute to the strangulation. Consequently, repeated mild fundic cholecystitis rather than ischemic cholecystitis may have developed.

In the previous two reports, the report of Ueo et al. [4] was very similar to our case. The clinical features and pathological findings of this case revealed repeated episodes of acute cholecystitis, indicating incomplete obstruction and strangulation of the gallbladder. The case of Miyakura et al. [5] was slightly different from ours, and the lesser omentum contributed to strangulation of the floating gallbladder, leading to acute ischemic cholecystitis. In this case, the lesser omentum was wrapped around the neck of the gallbladder and may have completely interrupted the blood supply and bile flow.

Establishing a preoperative diagnosis of a strangulated gallbladder by the omentum would be extremely difficult because the clinical symptoms, signs, and laboratory findings are similar to those of acute cholecystitis or torsion of the gallbladder. Abdominal ultrasonography (US) and computed tomography (CT) can facilitate early diagnosis of torsion of the gallbladder. Moreover, although US and CT can provide important information, such as the image of a gallbladder divided into two compartments. The wall of the neck compartment was normal, and the wall of the fundic compart was markedly thickened and edematous change, suggesting torsion of the fundus. In our patient, a narrowed and twisted area was seen between the edematous body and the normal neck of the gallbladder. Also, color Doppler sonography, which evaluates blood flow in the gallbladder wall, has been known to be helpful as a diagnostic tool. Cystic artery vascularity along the wall can be visualized in acute cholecystitis by color Doppler sonography, but blood flow is not detected in torsion of the gallbladder.

Strangulation of the floating gallbladder is rare and easily misdiagnosed because the clinical features and imaging findings of a strangulated gallbladder by the lesser omentum are similar to those of torsion of the gallbladder. An appropriate diagnosis and prompt surgical treatment are essential for a positive outcome.

\section{REFERENCES}

1. Losken A, Wilson BW, Sherman R. Torsion of the gallbladder: a case report and review of the literature. Am Surg 1997;63:975-8.

2. Wendel AV. VI. A case of floating gall-bladder and kidney complicated by cholelithiasis, with perforation of the gall-bladder. Ann Surg 1898;27: 199-202. 
3. Vedanayagam MS, Nikolopoulos I, Janakan G, El-Gaddal A. Gallbladder volvulus: a case of mimicry. BMJ Case Rep 2013;2013:bcr2012007857.

4. Ueo T, Yazumi S, Okuyama S, Okada Y, Oono T, Watanabe M, et al. Acute cholecystitis due to strangulation of a floating gallbladder by the lesser omentum. Abdom Imaging 2007;32:348-50.
5. Miyakura Y, Sadatomo A, Ohta M, Lefor AT, Sata N, Nishimura N, et al. Floating gallbladder strangulation caused by the lesser omentum: report of a case. Surg Today 2012;42:693-6.

6. Arslan ED, Hakbilir O, Uyanik B, Ozturk B, Kaya E, Ozturk D. Gallbladder volvulus. J Pak Med Assoc 2012;62:965-6. 